\title{
Analysis of Ivermectin as Potential Inhibitor of SARS-CoV-2 Using Resonant Recognition Model
}

\author{
Irena Cosic ${ }^{1}$, Drasko Cosic ${ }^{1}$, Ivan Loncarevic ${ }^{2}$
}

\author{
${ }^{1}$ AMALNA Consulting, Black Rock, 3193, Australia \\ ${ }^{2}$ QuantBioRes - QBR A/S, Copenhagen, 2860, Denmark
}

Running Title: Ivermectin as Potential Drug for COVID-19

\begin{abstract}
With worldwide spread of COVID-19 disease caused by SARS-CoV-2 virus, everyone is trying to find effective cure. One of the quickest approaches is to test existing already approved and safe drugs for possibility of their activity against SARS-CoV-2 virus. One of such possible drugs is ivermectin, which is FDA approved broad spectrum anti parasitic agent. Recently it has been shown that ivermectin has broad range of in vitro antiviral activities and it has been shown that ivermectin is an inhibitor of SARS-CoV-2. We have previously used our recently developed extended Resonant Recognition Model (RRM) for small molecules and we have proposed hat drugs like hydroxychloroquine, chloroquine and remdesivir can interfere with SARS-CoV-2 viral infection. Here, we have used an extended RRM model for small molecules to analyze the possibility for ivermectin, an already FDA-approved drug, to interfere with SARS-CoV-2 viral activity, which could lead to an effective cure.
\end{abstract}

Keywords: Drug Design, Small Molecules, Resonant Energy, Bioelectromagnetism, Resonant Recognition Model, SARS-Cov-2, COVID-19, Ivermectin

\begin{abstract}
Introduction
With the huge demand/development for new effective drugs there is large need for computational methods capable of quick preselection of potential drugs before they are chemically and biologically tested. With rapid development of biotechnology and specially pharmacology, there is busy time for drug developments. Most of these developments are based on trial and error, without thinking out of the box within biochemical experiments, which are time and resource consuming. So, there is large demand for computational rational drug preselection. The most of existing computational models are based on 3D and electrostatic fit (docking) between small molecule and related substrate. Such approaches are not considering general functionality of substrates with similar biological function, nor long distance specific recognition between small molecules and their substrates. Particularly, with the latest outbreak of coronavirus SARS-CoV-2 causing the COVID-19 pandemic, there is urgent need to preselect number of potential small molecule drugs that are already approved for other purposes and are clinically tested on a range of infected humans with a variety of success. The already established Resonant Recognition Model (RRM) proposes that selectivity of biological interactions and functions between proteins, DNA/RNA, is based on resonant electromagnetic energy between interacting
\end{abstract}

macromolecules at the specific frequency for the specific interaction/function. However, this approach cannot be applied for selective specific interactions between proteins and small molecules (potential drugs), as small molecules are not linear sequential molecules. Recently, we have extended the RRM model to small molecules interaction with proteins proposing that energy frequencies of free electrons in small molecules are the most relevant for their resonant recognition and interaction with proteins [1]. This extended RRM model has been tested for a couple of natural examples to explain and support the model. In addition, extended RRM model has been applied to already approved drugs, including remdesivir, chloroquine and hydroxychloroquine [1] and we have proposed that those small molecules are potential COVID-19 drugs. Another already approved safe drug, which has been tested for its activity against SARS-COV-2 virus is ivermectin, which we have analyzed here also using extended RRM model.

Ivermectin is FDA approved broad spectrum anti parasitic agent used to treat many types of parasite infestations [2]. Recently it has been shown that ivermectin has a broad range of in vitro antiviral activities [3-6]. Due to COVID-19 pandemic ivermectin has been also tested in vitro as potential inhibitor of SARS-CoV-2 replication and it has been

This article is published under the terms of the Creative Commons Attribution License 4.0

Author(s) retain the copyright of this article. Publication rights with Alkhaer Publications.

Published at: http://www.ijsciences.com/pub/issue/2021-01/

DOI: 10.18483/ijSci.2433; Online ISSN: 2305-3925; Print ISSN: 2410-4477 
shown that it indeed is an inhibitor of SARS-CoV-2 [7]. We have shown previously using our recently developed extended Resonant Recognition Model (RRM) for small molecules that drugs like hydroxychloroquine, chloroquine and remdesivir can interfere with SARS-CoV-2 viral infection [1]. Here, we have used extended RRM model for small molecules to analyze possibility for ivermectin, already FDA-approved drug, to interfere with SARSCoV-2 viral activity, which could lead to a cure.

\section{Methods}

\section{Extended Resonant Recognition Model for Small Molecules}

We have previously established Resonant Recognition Model (RRM) for analyzes of protein interactions with other proteins, DNA/RNA, which takes into account functionality of those macromolecules and is based on periodicities (frequencies) within distribution of free electron energies along these macromolecules [8-20]. With electron charge moving through protein, DNA/RNA backbone passing different free electron energies within different side groups (amino acids or nucleotides) electromagnetic radiation or absorption of frequency related to distribution of energies of free electrons along macromolecular backbone will be produced. The frequencies of these electromagnetic radiation or absorption depend on charge velocity, which is estimated to be at $7.87 \times 10^{5} \mathrm{~m} / \mathrm{s}$ [8-15]. For this velocity and the distance between amino acids in protein backbone, which is $3.8 \AA$, the frequency range obtained for protein interactions was estimated to be in the range of $10^{13} \mathrm{~Hz}$ up to $10^{15} \mathrm{~Hz}$. Therefore, the estimated range for both amino acid and nucleotide macromolecules includes far infra-red, infra-red and visible up to ultra-violet light spectrum. From the comparison with number of published experimental results [8-12,16-24], the strong linear correlation has been established between frequencies, as calculated using the RRM model and experimentally measured characteristic frequencies, with the slope factor of $\mathrm{K}=201 \quad[8-12,16-20]$. This correlation can be represented as following:

$\lambda=\mathrm{K} / \mathrm{f}_{\mathrm{rrm}}$

where $\lambda$ is the wavelength of light radiation in nanometres $(\mathrm{nm})$, which can influence particular biological process, $\mathrm{f}_{\mathrm{rrm}}$ is RRM numerical frequency and $\mathrm{K}$ is coefficient of this linear correlation. However, this already established RRM approach cannot be applied for interactions between proteins and small molecules, as small molecules are not linear sequential molecules. Still, we propose that small molecules also recognize proteins on the distance and interact with proteins through electromagnetic resonant energy transfer enabling specific biological activity. To expand the idea of electromagnetic resonant recognition to small molecules and their interaction with proteins, RRM model has been extended by calculating electromagnetic frequencies of free electron energies within the small molecule and comparing these frequencies with RRM characteristic frequencies for relevant interacting proteins [1].

For that purpose, we have proposed that energies of free electrons in small molecules are the most relevant for such resonant energy transfer and can be calculated as the electron-ion interaction pseudopotential (EIIP) of small molecule using the following semi-empirical formula as developed by Veljkovic [25-27]:

$<\mathrm{k}+\mathrm{q}|\mathrm{w}| \mathrm{k}>=0.25 \times \mathrm{Z} \times \sin (\pi \times 1.04 \times \mathrm{Z}) /(2 \times \pi)$

where $\mathrm{q}$ is change of momentum of delocalized electron in the interaction with potential $w$ (EIIP) in Rydberg $\left(\mathrm{Ry}=2.18 \times 10^{-18}[\mathrm{~J}]\right)$, while $\mathrm{Z}$ is average valence number over the whole small molecule.

The corresponding electromagnetic wavelength for this energy can be calculated using de Broglie formula as follows:

$\lambda=(\mathrm{h} \times \mathrm{c}) / \mathrm{E}$ in vacuum, where $\mathrm{c}$ is speed of light $\left(\mathrm{c}=2.998 \times 10^{8}[\mathrm{~m} / \mathrm{s}]\right)$

$\lambda=(\mathrm{h} \times \mathrm{v}) / \mathrm{E}$ in other materials, where $\mathrm{v}$ is speed of light in other materials

where $\lambda$ is wavelength of light $[\mathrm{nm}], \mathrm{h}$ is Planck constant $\left(\mathrm{h}=6.626 \times 10^{-34}[\mathrm{Js}]\right), \mathrm{E}$ is energy $[\mathrm{J}]$ of free electrons within small molecule.

All biological processes and interactions are taking place within biological materials and thus speed of light will depend on refraction index within biological materials:

$\mathrm{v}=\mathrm{c} / \mathrm{n}$

where $\mathrm{n}$ is refraction index of biological materials.

For water refraction index is 1.33, while for biological materials refraction index is: for cell membranes 1.46-1.60, for cytoplasm 1.35-1.39 and for proteins 1.36-1.55 [28].

Bearing in mind all the above, we have hypothesized that wavelengths (frequencies) produced by energies of free electrons within small molecules are critical for small molecule biological functions and their recognition and interaction with proteins and we have proposed that small molecules are recognizing and interacting with proteins through resonance at the 
same characteristic frequency (wavelength) [1]. To be able to compare resonant frequencies of small molecules, with resonant RRM frequencies, we can convert $\lambda_{b}[\mathrm{~nm}]$ into the RRM frequency, as related $\mathrm{RRM}$ frequency for small molecules can be presented using the formula below:

$\mathrm{f}_{\mathrm{sm}}=\mathrm{K}[\mathrm{nm}] / \lambda_{\mathrm{b}}[\mathrm{nm}]$

where $\mathrm{K}=201$ is coefficient in [nm] previously semiempirically identified to characterize the relationship between RRM frequencies and related electromagnetic radiation wavelengths, as explained above [8-11].

Thus, the RRM resonant frequency of small molecules can be calculated as follows:

$\mathrm{f}_{\mathrm{sm}}=(\mathrm{K} \times \mathrm{E}) /(\mathrm{h} \times(\mathrm{c} / \mathrm{n}))$

where: $f_{s m}$ is numerical RRM frequency corresponding to electromagnetic radiation resonances due to energies of free electrons within small molecules, $\mathrm{K}=201$ is coefficient in $\mathrm{nm}$ previously semi empirically identified to characterize the relationship between RRM frequencies and related electromagnetic radiation wavelengths, $\mathrm{E}$ is energy of free electrons within small molecules, $\mathrm{h}$ is Planck constant, $\mathrm{c}$ is speed of light, $\mathrm{n}$ is refraction index in biological materials.
The hypothesis, that wavelengths (frequencies) produced by energies of free electrons within small molecules are critical for small molecules biological functions and their recognition and interaction with proteins, has been already tested in couple of natural examples and some potential COVID-19 drugs [1].

\section{Results}

The first thing to do is identify and recognize SARS-CoV-2 spike proteins, which are on the surface of coronavirus [29]. We are proposing, based on our earlier experience with HIV virus [30-32], that all strains of SARS-CoV-2 spike proteins would have one common component that characterize their recognition and interaction with host cells. To find out this characteristic component we have utilized the RRM model, which is capable to analyze functional characteristics, as common periodicities (frequencies) for all proteins with the same function/recognition. When we have applied the RRM analysis to spike proteins from different coronaviruses, from UniProt database, as listed in Appendix, we have found the common component that can characterize the spikes recognition and interaction with host cells at the most prominent common RRM frequency of $0.2827+0.0009$, as presented in Figure

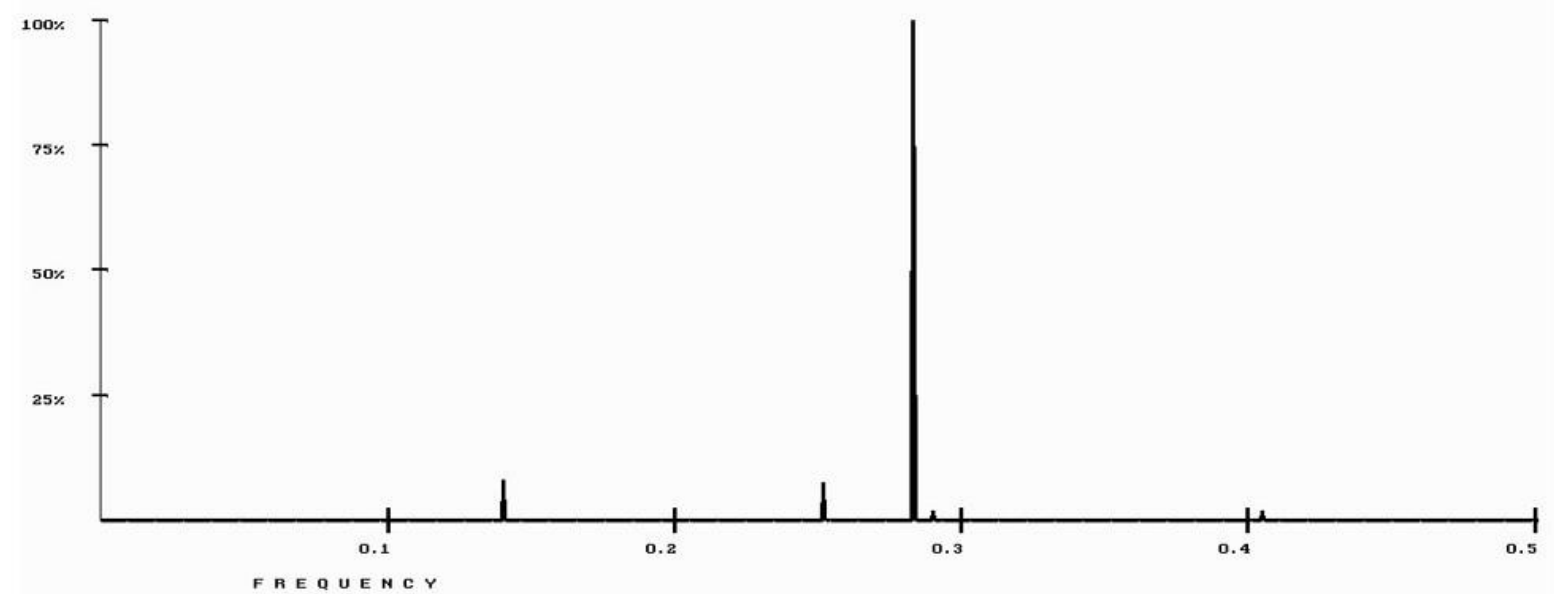

Figure 1. RRM cross-spectrum of spike proteins. The common RRM characteristic frequency is at $0.2827 \pm 0.0009$.

It is interesting to note that there is one unique common characteristic for all analyzed spike proteins from many different coronaviruses. This would mean that all coronaviruses have one and the same RRM characteristic frequency at $0.2827 \pm 0.0009$, characterizing viral recognition and interaction with host cells. Thus, there is a possibility to interfere with this frequency and prevent the very first step of viral infection by using small molecule drugs. One of such candidates is ivermectin, which has been already approved for treatment of parasites and has already shown to inhibit replication of SARS-CoV-2 in vitro [7].

There are two variants of ivermectin: ivermectinB1A with chemical formula $\left(\mathrm{C}_{48} \mathrm{H}_{74} \mathrm{O}_{14}\right)$ and ivermectinB1B with chemical formula 
$\left(\mathrm{C}_{47} \mathrm{H}_{72} \mathrm{O}_{14}\right)$.

Using the chemical formula for ivermectinB1A small molecule $\left(\mathrm{C}_{48} \mathrm{H}_{74} \mathrm{O}_{14}\right)$, we have calculated, as described in Methods section, energy of free electrons within ivermectinB1A small molecule to be at $\mathrm{Ea}=0.0870 \mathrm{Ry}$. For this energy and refraction index for proteins, which is between 1.36-1.55 [28], we have calculated the corresponding RRM frequency for ivermectinB1A small molecule to be between 0.26-0.30, as highlighted in green in Figure

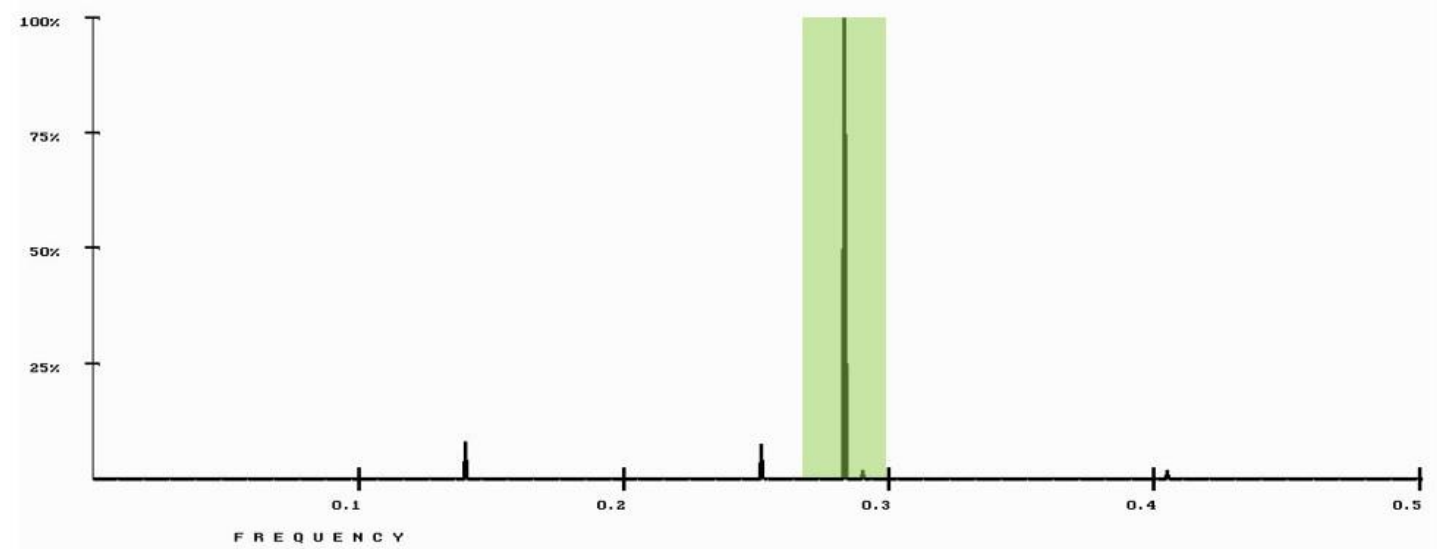

Figure 2. RRM cross-spectrum of spike proteins. The common RRM characteristic frequency is at $0.2827 \pm 0.0009$. RRM frequency range for ivermectinB1A small molecule between 0.26-0.30 highlighted in green.

It is important to notice that this RRM frequency range for ivermectinB1A small molecule overlaps the characteristic RRM frequency for SARS-CoV2 spike proteins, which is at $0.2827 \pm 0.0009$. As there is an overlap between characteristic RRM frequency for coronavirus spike proteins including SARS-CoV-2 and RRM frequency for ivermectinB1A small molecules, we propose that ivermectinB1A could interact with SARS-CoV-2 spike proteins and could be efficient in the first phase of infection by neutralising activity of spike proteins on the surface of coronavirus and thus preventing initial viral infection. It is also interesting to note that range of RRM characteristic frequencies for ivermectinB1A is exactly the same as previously calculated [1] for hydroxychloroquine indicating that although these two molecules have different chemical formulas they can have the same biological activity.

Using the chemical formula for ivermectinB1B small molecule $\left(\mathrm{C}_{47} \mathrm{H}_{72} \mathrm{O}_{14}\right)$, we have calculated, as described in Methods section, energy of free electrons within ivermectinB1B small molecule to be at $\mathrm{Eb}=0.0852 \mathrm{Ry}$. For this energy and refraction index for proteins, which is between 1.36-1.55 [28], we have calculated the corresponding RRM frequency for ivermectinB1B small molecule to be between 0.25-0.29, as highlighted in green in Figure

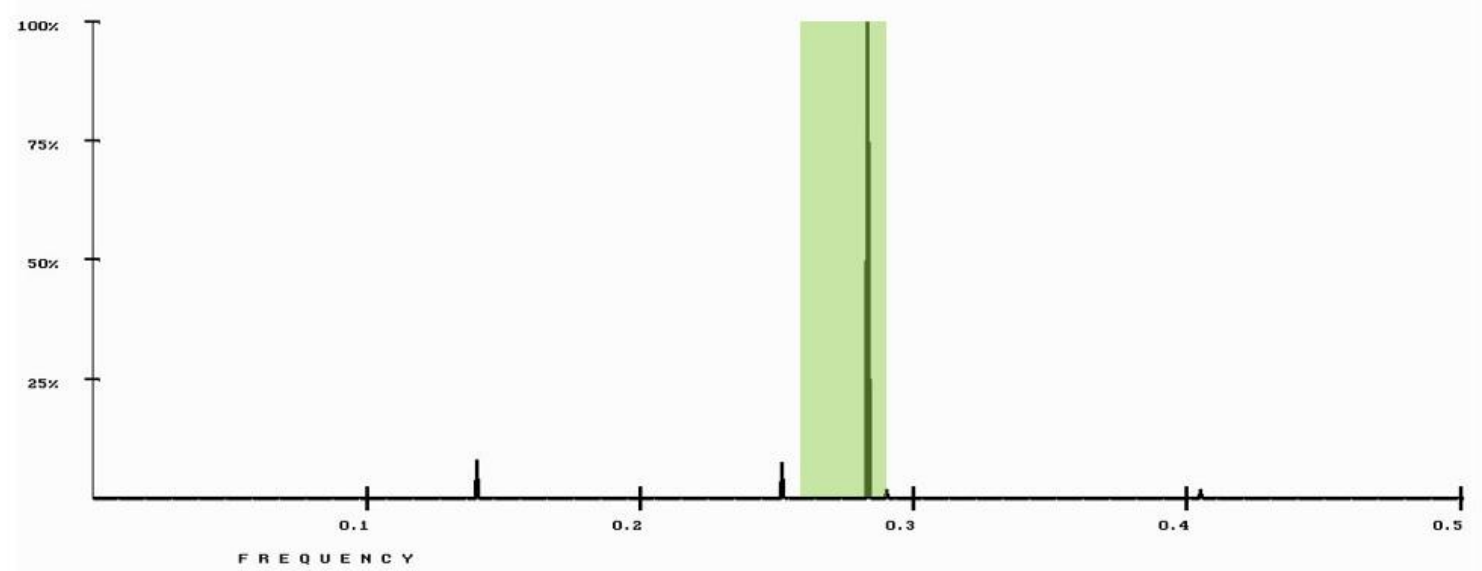

Figure 3. RRM cross-spectrum of spike proteins. The common RRM characteristic frequency is at $0.2827 \pm 0.0009$. RRM frequency range for ivermectinB1B small molecule between 0.25-0.29 highlighted in green. 
characteristic RRM frequency for SARS-CoV-2 spike proteins, which is at $0.2827 \pm 0.0009$. As there is overlap between characteristic RRM frequency for coronavirus spike proteins including SARS-CoV-2 and RRM frequency for ivermectinB1B small molecules, we propose that ivermectinB1B could interact with SARS-CoV-2 spike proteins and thus could be efficient in the first phase of infection by neutralising activity of spike proteins on the surface of coronavirus and thus preventing initial viral infection.

\section{Discussion and Conclusion}

Previously, we have proposed a completely new approach for preselection of bioactive drugs (small molecules) based on electromagnetic energy resonances between small molecules and substrates/proteins considering long distance selective resonance, as well as common biological functionality of related substrates/proteins [1]. This extended RRM model opens new horizons for biochemistry and pharmaceutical industry in analyzes of small molecule - protein interaction, consequently in preselection of new drugs and drug design in general.

We have previously presented two natural examples of small molecules interactions with related receptors, namely: cannabinoids with cannabinoid receptors and kainic acid (kainate) with glutamate kainic receptors. In addition, we presented application to already approved drugs, which are also potential COVID-19 drugs including remdesivir, chloroquine and hydroxychloroquine for their ability to interact with viral proteins and thus interfere with viral infection [1]. These examples have shown that electromagnetic frequencies of free electrons within the small molecules are overlapping the characteristic RRM frequencies of related receptors/proteins, indicating that interactions between small molecules and related receptors/proteins are indeed based on resonant electromagnetic energy at a specific frequency for a specific interaction.

Here, we have applied the extended RRM model to ivermectin with the aim to find out if ivermectin is potential candidate to interact and possibly inhibit SARS-CoV-2's activity. During this analysis we have found that the RRM frequency corresponding to energy of free electrons within ivermectin corresponds to RRM frequency characterizing coronavirus spike proteins including SARS-CoV-2. Thus, we can propose that ivermectin could interfere with activity of spike proteins and as such could be efficient treatment for the first phase of SARS-CoV-2 infection by neutralising activity of spike proteins on the surface of coronavirus and preventing initial viral infection, in the similar way as for hydroxychloroquine [1].

Our findings are in complete agreement with experimental results, which have shown that ivermectin has ability to inhibit SARS-CoV-2 virus [7]. We have shown here that the extension of the RRM model, which is aimed generally to aid developments within pharmaceutical industry, can satisfy demand for pre-selective computational methods capable of quick preselection of small molecules before they are chemically and biologically tested. The extended RRM model, which represents quick preselection method that could significantly narrow down a number of experimentally tested potential molecular candidates is capable to enormously save resources, time and costs that are involved in discovery of new drugs, and could provide quicker and better health outcomes.

Consequently, the extended RRM model is opening new horizons for, not only understanding protein, DNA/RNA selectivity of interactions, but also for understanding selectivity of interactions between small molecules and related receptors/proteins. This could lead to new directions for pharmaceutical industry, drug design and biochemistry in general.

\section{Contributions}

Conceptualization, I.C.; Methodology, I.C. and D.C.; Software, D.C.; Resources I.L.; WritingOriginal Draft Preparation-Review and Editing, I.C., D.C. and I.L.

\section{Competing Interests}

Authors declare they have no competing interests.

\section{Funding}

This research received no external funding.

\section{Acknowledgement}

The authors would like to thank Miss Amy Cosic for editing this manuscript and Mr. Anthony Slingsby for proofreading this manuscript.

\section{References}

1. Cosic I, Cosic D, Loncarevic I: New Concept of Small Molecules Interaction with Proteins - An Application to Potential COVID-19 Drugs, International Journal of Sciences, 2020, 9(9), 16-25, doi: 10.18483/ijSci.2390.

2. Gonzales Canga A, Sahagun Prieto AM, Diez Liebana MJ, Fernandez Martinez N, Sierra Vega M, Garcia Vieitez JJ: The Pharmacokinetics and Interactions of Ivermectin in Humans - A Mini-Review. AAPS J, 2008; 10(1), 42-46.

3. Gotz V, Magar L, Dornfeld D, Giese S, Pohlmann A, Hoper D, Kong BW, Jans DA, Beer M, Haller O, Schwemmle M: Influenza A Viruses Escape from MxA Restriction at the Expense of Efficient Nuclear vRNNP Import. Scientific 
Reports, 2016; 6, 23138

4. Lundberg L, Pinkham C, Baer A, Amaya M, Narayanan A, Wagstaff KM, Jans DA, Kehn-Hall K: Nuclear Import and Export Inhibitors Alter Capsid Protein Distribution in Mammalian Cells and Reduce Venezuelan Equine Encephalitis Virus Replication. Antiviral Research, 2013; 100(3), 662-672, doi: 10.1016/j.antiviral.2013.10.004.

5. Tay MYF, Fraser JE, Chan WKK, Moreland NJ, Rathore AP, Wang C, Vasudevan SG, Jans DA: Nuclear Localization of Dengue Virus (DENV) 1-4 Non-structural Protein 5, Protection Against All 4 DENV Serotypes by the Inhibitor Ivermectin. Antiviral Research, 2013; 301-306, doi: 10.1016/j.antiviral.2013.06.002.

6. Wagstaff KM, Sivakumaran H, Heaton SM, Harrich D, Jans DA: Ivermectin is a Specific Inhibitor of Importin $\alpha / \beta$ mediated Nuclear Import Able to Inhibit Replication of HIV1 and Dengue Virus. Biochemical Journal, 2012; 443(3), 851-856.

7. Caly L, Druce JD, Catton MG, Jans DA, Wagstaff KM: The FDA-approved Drug Ivermectin Inhibits the Replication of SARS-CoV-2 in vitro. Antiviral Research, 2020; 178, 104787 doi: 10.1016/j.aniviral.2020.104787.

8. Cosic I: Macromolecular Bioactivity: Is it Resonant Interaction between Macromolecules? -Theory and Applications. IEEE Trans on Biomedical Engineering, 1994; 41, 1101-1114.

9. Cosic I: The Resonant Recognition Model of Macromolecular Bioactivity: Theory and Applications. Basel: Birkhauser Verlag, 1997.

10. Cosic I: Resonant Recognition Model of Protein-Protein and Protein-DNA Recognition, in Bioinstrumentation and Biosensors. Marcel Dekker Inc New York, 1990; 475-510.

11. Cosic I, Paspaliaris V, Cosic D: Biophysical Insights into Cystic Fibrosis Based on Electromagnetic Resonances in CFTR Proteins, International Journal of Sciences, 2019; 8(9), 1-8, doi: 10.18483/ijSci.2148.

12. Cosic I, Lazar K, Cosic D: Cellular Ageing - Telomere, Telomerase and Progerin analysed using Resonant Recognation Model. MD-Medical Data, 2014; 6(3), 205-209.

13. Cosic I, Cosic D, Lazar K: Analysis of Tumor Necrosis Factor Function Using the Resonant Recognition Model. Cell Biochemistry and Biophysics, 2015; doi: 10.1007/s12013015-0716-3.

14. Cosic I, Paspaliaris V, Cosic D: Analysis of Protein-Receptor on an Example of Leptin-Leptin Receptor Interaction Using the Resonant Recognition Model. Appl. Sci., 2019; 9, 5169, doi: 10.3390/app9235169.

15. Cosic I, Cosic D, Loncarevic I: RRM Prediction of Erythrocyte Band3 Protein as Alternative Receptor for SARS-CoV-2. MDPI Appl. Sci., 2020; 10, 4053, doi: 10.3390/app10114053.

16. Cosic I, Lazar K, Cosic D: Prediction of Tubulin Resonant Frequencies Using the Resonant Recognition Model (RRM). IEEE Trans. on NanoBioscience, 2015; 12, 491-496; doi: 10.1109/TNB.2014.2365851

17. Cosic I, Cosic D, Lazar K: Is It Possible to Predict Electromagnetic Resonances in Proteins, DNA and RNA? Nonlinear Biomedical Physics, 2015; 3, doi: 10.1140/s40366015-0020-6.

18. Cosic I, Cosic D: The Treatment of Crigler-Najjar Syndrome by Blue Light as Explained by Resonant Recognition Model. EPJ Nonlinear Biomedical Physics, 2016; 4(9), doi: 10.1140/epjnbp/s40366-016-0036-6.

19. Cosic I, Cosic D, Lazar K: Environmental Light and Its Relationship with Electromagnetic Resonances of Biomolecular Interactions, as Predicted by the Resonant Recognition Model. International Journal of Environmental Research and Public Health, 2016; 13(7), 647, doi: 10.3390/ijeprh13070647.
20. Cosic I, Paspaliaris V, Cosic D: Explanation of Osteoblastic Differentiation of Stem Cells by Photo Biomodulation Using the Resonant Recognition Model. Appl. Sci., 2019; 9, 1979 , doi: 10.3390/app9101979.

21. Vojisavljevic V, Pirogova E, Cosic I: The Effect of Electromagnetic Radiation (550nm-850nm) on I-Lactate Dehydrogenase Kinetics. Internat J Radiat Biol, 2007; 83, 221-230.

22. Dotta BT, Murugan NJ, Karbowski LM, Lafrenie RM, Persinger MA: Shifting Wavelength of Ultraweak Photon Emissions from Dying Melanoma Cells: Their Chemical Enhancement and Blocking Are Predicted by Cosic's Theory of Resonant Recognition Model for Macromolecules. Naturwissenschaften, 2014; 101(2), doi: 10.1007/s00114013-1133-3.

23. Murugan NJ, Karbowski LM, Persinger MA: Cosic's Resonance Recognition Model for Protein Sequences and Photon Emission Differentiates Lethal and Non-Lethal Ebola Strains: Implications for Treatment. Open Journal of Biophysics, 2014; 5, 35.

24. Karbowski LM, Murugan NJ, Persinger MA: Novel Cosic Resonance (Standing Wave) Solutions for Components of the JAK-STAT Cellular Signalling Pathway: A Convergence of Spectral Density Profiles. FEBS Open Bio, 2015; 5, 245-250.

25. Veljkovic V, Slavic I: General Model od Pseudopotentials. Physical Review Letters, 1972; 29, 105-108.

26. Veljkovic V: A Theoretical Approach to Preselection of Cancerogens and Chemical Carcinogenesis. Gordon \& Breach New York, 1980.

27. Veljkovic V: The Dependence of the Fermi Energy on the Atomic Number, Physics Letters, 1973; 45A(1), 41-42.

28. Mohsin ASM, Salim MB: Probing the Intracellular Refractive Index and Molecular Interaction of Gold Nanoparticles in HeLa Cells Using Single Particle Spectroscopy. International Journal of Nanomedicine, 2018 13, 6019-6028, doi: 10.2147/IJN.S175523.

29. Li F: Receptor Recognition and Cross-species Infections of SARS Coronavirus. Antiviral Research, 2013; 100(1), 24654, doi: 10.1016/j.antiviral.2013.08.014.

30. Krsmanovic V, Biquard JM, Sikorska-Walker M, Cosic I, Desgranges C, Trabaud MA, Whitfield JF, Durkin JP, Achour A, Hearn MT: Investigation Into the Cross-reactivity of Rabbit Antibodies Raised against Nonhomologous Pairs of Synthetic Peptides Derived from HIV-1 gp120 proteins. J.Peptide Res, 1998; 52(5), 410-412.

31. Hearn MTW, Biquard JM, Cosic I, Krsmanovic V: Peptides Immunologically related to proteins expressed by a vira agent, having a sequence of amino acids ordered by means of protein informational method. US Patent 6, 294, 174, 2001.

32. Achour A, Biquard JM, Krsmanovic V, M'Bika JP, Ficheux D, Sikorska M, Cozzone AJ: Induction of Human Immunodeficiency Virus (HIV-1) Envelope Specific CellMediated Immunity by a Non-Homologus Synthetic Peptide. PLoS ONE, 2007; 11, 1-12, doi: 10.1371/journal.pone.0001214.

\section{Appendix}

The protein sequences from UniProt Database analyzed using the RRM model:

Cleaved, active spike proteins: K9N5Q8, Q5MQD0, Q14EB0, Q0ZME7, P36334, P59594,

P11224, P11225, Q8BB25, Q9IKD1, P05135, P11223, Q0Q466, P36300, Q65984, Q7T6T3, P15423, Q6Q1S2, P18450, P33470, P07946, P27655, P24413, Q01977, P10033, Q91AV1 and QHD43416.1. 